\title{
miRNAs regulate immune response and signaling during hepatitis $C$ virus infection
}

Huange Zhu*, Yan Geng, Qian He and Miaoxian Li

\begin{abstract}
Hepatitis $C$ is one of the most common types of viral hepatitis that impair human health. At present, there is still no effective specific therapy for hepatitis C virus infection. As host immunity is an important mechanism to defend against or clear infections, the interactions between the virus and the host immune response are crucial to the progress of the disease. Of note, hepatitis C virus infection has been reported to regulate cellular miRNAs, which play significant roles in many processes, including infection and immunity. In this review, we describe how miRNAs regulate the host immune response to hepatitis $C$ virus via complex signaling pathways.
\end{abstract}

Keywords: Hepatitis C virus (HCV), miRNA, Immune response, Signaling pathway

\section{Background}

Hepatitis $\mathrm{C}$ virus ( $\mathrm{HCV})$, an RNA virus in the family Flaviviridae, causes one of the most common types of viral hepatitis. Remarkably, the incidence of viral hepatitis continues to increase, despite progress in controlling many other infectious diseases. The primary modes of HCV transmission are unsafe blood transfusion, injection drugs abuse, and maternal-neonatal. Like other viruses, $\mathrm{HCV}$ provokes innate and adaptive immune responses that enable the host to resist infection. When such responses fail, the virus replicates and induces acute or chronic hepatitis, as seen both in patients infected with $\mathrm{HCV}$ and hepatitis B virus (HBV). Although both $\mathrm{HCV}$ and $\mathrm{HBV}$ infection induce acute and chronic liver disease, they have profoundly different natural histories and outcomes. Vertical transmission of HBV from mother to neonate always results in chronic hepatitis; instead, HBV infection during adulthood typically results in lifelong protective immunity [1]. By contrast, $\mathrm{HCV}$ infection readily establishes chronic hepatitis in $60-80 \%$ of infected adults [1]. Today, there are about 360 million and 180 million cases of chronic hepatitis B and C

*Correspondence: hgzhu2012@163.com

Clinical Laboratory of The Second Affiliated Hospital of Xi'an Jiaotong

University (Xibei Hospital), \#157 Xiwu Road, Xincheng District,

Xi'an 710004, China infection, respectively [2]. Due in part to impaired immunity, HCV infection is more likely to progress to chronic hepatitis $\mathrm{C}$, a leading cause of liver cirrhosis and, subsequently, hepatocellular carcinoma. In chronic hepatitis C, $\mathrm{HCV}$ can co-exist with a permanently host immune system for decades. Indeed, about $80 \%$ of hepatocellular carcinoma cases are related to chronic virus infection [2]. In turn, hepatocellular carcinoma is the most common form of liver cancer, and accounts for the sixth and third largest cases of cancer morbidity and lethality in the world, highlighting the significance of $\mathrm{HCV}$ as a worldwide public health issue.

The traditional therapy for $\mathrm{HCV}$ infection is a combination of interferon (IFN) and ribavirin. However, this regimen has low cure rate, and is effective only for a fraction of patients [3]. On the other hand, direct-acting anti-viral, which is still considered as a new therapeutic approach, has a higher cure rate [4], but high cost and drug resistance have limited wide use. Hence, there is still no effective specific therapy that can be widely used against HCV. Since the host immunity is vital in the process of $\mathrm{HCV}$ infection, analysis of the host immune response is crucial to identify new targets for therapy, as well as to understand the specific mechanisms driving $\mathrm{HCV}$ infection.

Of note, expression or dysregulation of microRNAs (miRNAs) are associated with an array of disease states, 
including diabetes, neurological, blood, and immune disorders $[5,6]$. miRNAs are small noncoding RNAs, which, along with long noncoding RNAs, comprise a class of RNAs that do not encode proteins. Mature miRNAs are about 18-25 nucleotides, and are generated via RNA polymerase II/III-dependent transcription and subsequent processing by RNase III [7]. In 1993, Ambros and collaborators demonstrated that the miRNA lin4 inhibits LIN14 protein expression, but not mRNA expression [8]. Since then, miRNAs have become the focus of extensive mechanistic and functional studies.

miRNAs play important roles in growth and development. For instance, some miRNAs are specifically expressed in embryonic stem cells, and probably help maintain pluripotency [9]. miRNAs also mediate late embryo gastrulation, heart development, neurogenesis, and other events [10]. In addition, miRNAs modulate metabolism by regulating insulin release, cholesterol metabolism, and other processes. Finally, miRNAs regulate the expression of target mRNAs at transcription or post-transcription, such as by inhibiting translation or by altering mRNA stability.

\section{miRNAs regulate the immune response to $\mathrm{HCV}$}

Organisms are constantly exposed to pathogens in the environment, which are subsequently cleared by the host's innate and acquired immunity. Although innate immunity is the first line of defence against pathogens, acquired immune responses are also necessary for full protection. As such, HCV has evolved escape strategies to establish persistent infections. Chronic hepatitis, cirrhosis, hepatocellular carcinoma, and other diseases associated with $\mathrm{HCV}$ are closely linked to the host immune response [11]. On the other hand, a growing body of evidence indicates that miRNAs shape infection and immunity $[6,12]$, not only by regulating the differentiation and development of immune cells, but also by mediating the immune response to infection or other disorders. For example, miRNAs suppressed by $\mathrm{HCV}$ regulate immune reactions, antigen presentation, cell cycle progression, and lipid metabolism, while miRNAs suppressed by hepatitis B virus regulate cell death, DNA damage and repair, and transcription [13].

The miRNA miR-122 is specifically expressed in the liver, and accounts for approximately $70 \%$ of total liver miRNA [14]. miR-122 binds to the $5^{\prime}$-UTR of and stabilizes the HCV RNA genome, and thereby stimulates virus replication [14, 15]. On the other hand, pre-miR-122 or unprocessed single-stranded miR-122 cannot bind to the HCV 5'-UTR, and cannot modulate HCV RNA accumulation. It is essential for miR-122 modulation of $\mathrm{HCV}$ translation and RNA accumulation, that premiR-122 must be processed by Dicer and TRBP and strand selection of mature duplex miR-122 [16]. Human Dicer and TRBP proteins involve in the biogenesis pathway of miR-122 mature, but they are not needed for the mechanism of HCV RNA accumulation and HCV translation provided by mature duplex miR-122 [16]. Inhibition of miR-122 also boosts HO-1 mRNA expression and reduces HCV RNA expression, highlighting its potential as a therapeutic target [17]. An inverse correlation between miR-122 and biochemical evidences of hepatocyte damage, including alanine aminotransferase, aspartate aminotransferase, and fibrosis, but not viral load, has also been noted in patients with chronic hepatitis C [18], with miR-122 expression consistently downregulated at advanced stages of fibrosis. Decreased levels of miR-122 were also predictive of poor response to therapy with IFN [19]. Strikingly, miR-122 was also shown to impede HCV entry into hepatocytes by binding the $3^{\prime}$-UTR of OCLN mRNA. Accordingly, miR-122 mimics may be more beneficial than miR-122 inhibitors in early infection [20], as low miR-122 levels at the beginning of infection may facilitate HCV entry into hepatocytes. Both miR-122 inhibitors and mimics have advantages in HCV infection, which still needs to have further careful studies to evaluate.

Various relationships between miR-122 and hepatocellular carcinoma have also been noted. Some studies showed that miR-122 is strongly expressed in malignant liver nodules, and may promote tumorigenesis by inhibiting tumour suppressors in hepatocellular carcinoma related to HCV infection [21]. Corollary, other studies indicated that miR-122 is downregulated in hepatocellular carcinoma unrelated to HCV [22]. Collectively, these studies imply that miR-122 plays essential and complex roles not only in HCV infection, but also in hepatocellular carcinoma regardless of HCV status [21].

miR-155, an miRNA linked to inflammatory monocyte activation and a common target of many inflammatory mediators, not only promotes autoimmune inflammation by enhancing the development of inflammatory T-cells [23], but also plays important roles in immune response and HCV infection [24]. For instance, HCV induces miR155 expression in vitro and in vivo. Accordingly, miR155 is significantly more abundant in the liver tissue and serum in HCV-infected patients than in uninfected controls $[25,26]$. In addition, miR- 155 markedly diminishes in patients who successfully clear the virus after treatment, reinforcing the notion that upregulated miR-155 facilitates HCV infection [25]. Indeed, miR-155 regulates the innate immune response by regulating IFNr production from natural killer cells during chronic hepatitis $\mathrm{C}$ infection [27], and controls the immune response to $\mathrm{HCV}$ in the liver [24]. 
Similar to miR-155, miR-146a is abundant in peripheral blood mononuclear cells and regulates the inflammatory and immune responses [28]. It has recently been reported that miR-146 was upregulated in HCV-infected cells, human primary hepatocytes and liver tissue of chronic hepatitis $C$ infection patients [28]. On the other hand, decreased miR-146a expression has also been observed in peripheral blood mononuclear cells from patients with chronic hepatitis $C$. This can partially be explained as the studies analysed patients infected with different genotype HCV. Moreover, miR-146a expression is significantly correlated to serum cholesterol, and well as cholesterol levels in peripheral blood mononuclear cells. This is not unsurprising as the cholesterol metabolism plays a critical role in the HCV life cycle. However, the detail mechanisms in miR-146a expression and cholesterol metabolism, and its inflammatory and immune roles remain to be elucidated.

The role of miR-130a in HCV infection is similarly complex. miR-130a expression in the $\mathrm{HCV}$-infected liver is much higher than in control, and miR-130a knockdown also inhibits HCV RNA replication in hepatocytes by inducing IFITM1 expression [29], which, in turn, stimulates secretion of IFN. Thus, HCV may persist by up-regulating miR-130a to inhibit IFITM1 and the subsequent innate immune response. On the other hand, miR130a overexpression was also reported to suppress $\mathrm{HCV}$ RNA replication in both the Con $1 b$ replicon and in the JFH1-based cell culture system [30]. Further, miR-130a was found to downregulate miR-122 but upregulate proteins that coordinate the host innate immune response, including type I IFN (IFN $\alpha /$ IFN $\beta$ ), ISG15, USP18, and MxA. Collectively, the data indicate that miR-130a may play dual roles in HCV replication by shaping the host innate immune response.

miR-21 is also upregulated both in liver biopsies and Huh7.5 cells after HCV infection (18). In turn, miR-21 represses the expression of type I IFN and the subsequent anti-viral response to enable the virus to evade the host immune system and replicate [31]. miR-21 also stimulates the proliferation of hepatocellular carcinoma cells [32], perhaps driving disease progression in this manner. Indeed, miR-21 is positively correlated with clinical indices in patients with chronic hepatitis $\mathrm{C}$, including viral load, fibrosis, serum alanine aminotransferase, and aspartate aminotransferase [18]. Moreover, miR21 directly targets SMAD7, a member of SMADs which was described in details follow-up, in Huh7 cells [18], although the pathologic significance of this relationship is unclear.

Dual specific phosphatases (DUSP) are signature markers of $T$ cell aging, of which DUSP 5 and 6 have been implicated in loss of $\mathrm{T}$ cell antigen receptor sensitivity [33]. Remarkably, a drop in miR-181a expression in $\mathrm{CD}_{4}^{+}$
$\mathrm{T}$ cells derived from HCV-infected patients boosts DUSP 6 expression and $\mathrm{CD}^{+}{ }^{+} \mathrm{T}$ cell dysfunction [34], suggesting a possible mechanism by which HCV interacts with the cellular immune response to establish infection.

Lipid overload due to HCV infection also boosts expression of miR-27 in the liver, which, in turn, inhibits virus infection and promotes lipid storage. However, this suppressive effect also enables the virus to escape immune surveillance by lowering the viral load [35].

By directly binding the $3^{\prime}$-UTR of Bach1 mRNA, miR196 suppresses Bach1 expression, stimulates HMOX1 expression, and inhibits HCV gene expression [36]. Surveys showed that circulating miR-196a is significantly lower in patients with chronic hepatitis $\mathrm{C}$, regardless of $\mathrm{HCV}$ viral load or alanine aminotransferase levels. This result was attributed to the reduced release of miR-196a from HCV-infected hepatocytes, highlighting miR-196a as a potential biomarker for early diagnosis of chronic hepatitis C [37]. Of note, miR-196a expression can be stimulated in hepatocellular carcinoma cells by exposure to IFN $\beta$ [38]. Finally, we note that many other miRNAs and miRNA clusters have been reported to impact $\mathrm{HCV}$ infection.

\section{Signaling pathways regulated by miRNAs during HCV infection}

Signaling pathways, which form a highly complex crossregulated network in cells, are the mechanism by which extracellular molecules transmit signals into cells, trigger a series of enzymatic and biochemical reactions, and ultimately elicit cellular responses. These pathways are tightly regulated as aberrant signaling can have pathophysiological consequences. Accordingly, the various interactions between miRNAs and $\mathrm{HCV}$ are closely linked to these pathways.

\section{Toll-like receptor (TLR) signaling}

TLR signaling activates the innate immunity and promotes the development of antigen-specific acquired immunity. There is more than one receptor involved in TLR signaling. TLRs are a family of receptor members, a type of pattern recognition receptors (PRRs). These receptors are widely expressed on the membranes of immune and non immune cells, including dendritic cells, macrophages, natural killer cells and fibroblasts, which is crucial for recognizing or defensing invading pathogens and endogenous ligands. Ligand binding triggers the recruitment and modification of downstream signaling molecules-including MyD88, IRAKs, TAK1, TAB1, TAB2, and TRAF6 - to ultimately induce NF- $\mathrm{kB}$ nuclear translocation and regulate target gene expression.

$\mathrm{HCV}$ infection upregulates miR-21 expression and miR-21 suppresses the production of type I IFN [31]. 
Moreover, miR-21 overexpression and inhibition in Huh7 hepatocytes can decrease and increase the expression of several TLR pathway effectors, including MyD88, IRAK1, IRAK4, and TRAF6- of which MyD88 and IRAK1 are direct targets of miR-21 and can modulate type I IFN production. Thus, HCV infection upregulates miR-21 and represses IFN $\alpha$ signaling through MyD88 and IRAK1 [31], both of which link TLR and IL-1R to downstream signaling molecules. In turn, repression of type I IFN helps the virus evade the host immune system, suggesting that TLR signaling is one mechanism by which miRNAs can regulate virus invasion. While these founds of miR21 are studied in hepatocytes which are less TLR expressers than Kupffer cells, the complex roles and mechanisms still need further studies. In addition, there is an inverse correlation between miR-125b levels and cytokine expression after HCV core protein challenge [39]. Studies have shown that miR-125b expression is downregulated and cytokine production was upregulated in THP-1 cells, a human monocytic leukemia cell line, following HCV core protein stimulation. Furthermore, forced miR-125b expression can abolish the cytokine production induced by $\mathrm{HCV}$ core protein by inhibiting NF-kB p65, ERK, and p38 phosphorylation. Collectively, this evidence suggests that miR-125b may negatively regulate $\mathrm{HCV}$-induced immune responses by targeting TLR2/MyD88 signaling in monocytes [39]. And it still should be noticed that forced $\mathrm{HCV}$ core protein expression may not recapitulate the exact process and mechanism during $\mathrm{HCV}$-infection. These works give us new sight and information that may be crucial for HCV infection, which still need further studies to verify.

\section{JAK/STAT signaling}

The Janus kinase/signal transducer and activator of transcriptions (JAK/STAT) pathway is frequently engaged by cytokines and growth factors that regulate cell proliferation, differentiation, apoptosis, inflammation, and other processes. Indeed, IFN activates this pathway to elicit an anti-viral response and JAK/STAT pathway activation may lead to higher IFN therapy responses. On the other hand, JAK/STAT signaling is suppressed by suppressors of cytokine signaling (SOCS) proteins, of which SOCS3 is one of the most potent. Therefore, both JAK/STAT signaling activity and SOCS proteins may play functional roles in IFN resistance in $\mathrm{HCV}$-infected patients.

Notably, silencing miR-122, which is strongly expressed upon $\mathrm{HCV}$ infection and is predictive of the response to IFN therapy [14, 19], also suppresses SOCS3 expression by methylation of its promoter, and thereby boosts STAT3 activation [40] to enhance the response to IFN therapy. miR-19a, a member of the miR-17-92 cluster that is dysregulated in $\mathrm{HCV}$ infection [41], probably has similar clinical value, as it suppresses SOCS3 expression to enhance IFN $\alpha$ and interleukin-6 signaling via STAT3, and thus activates JAK/STAT signaling [42]. HCV infection also stimulates miR-373 expression, and thereby impairs JAK/STAT signaling by directly targeting JAK1 and IFN-regulating factor 9 [43]. Taken together, these observations imply that JAK/STAT signaling and SOCS proteins may underlie IFN resistance in $\mathrm{HCV}$ patients.

\section{TGF- $\beta 1 / S M A D s$ signaling}

Transforming-growth factor- $\beta$ (TGF- $\beta$ ) is synthesized by many cells and has a fibrogenic capacity by serving as a chemotactic for fibroblasts, stimulating fibroblast proliferation, and increasing extracellular matrix protein synthesis. In addition, TGF- $\beta$ is critical for liver fibrogenesis induced by virus infection or other factors, and also has anti-inflammatory and immunosuppressive effects upon binding its cognate receptors.

SMADs are a family of intracellular regulatory proteins that regulate signaling downstream of TGF- $\beta$ receptors. SMADs can be divided into three classes, including receptor-regulated SMADs, common-mediator SMAD and inhibitory SMADs. SMAD7 is one inhibitory SMAD, which can block the activation of receptor-regulated SMADs and common-mediator SMADs. Notably, upregulation of miR-21 in HCV and hepatocellular carcinoma patients represses a luciferase reporter containing the $3^{\prime}$-UTR of SMAD7 and increases luciferase production from a TGF- $\beta$ reporter [18]. On the other hand, SMAD7 is a feedback inhibitor of TGF- $\beta$ and its expression is induced by TGF- $\beta$. Collectively, these observations highlight a potential relationship between miR-21 and TGF$\beta 1$, possibly via SMAD7, that could drive fibrogenesis in chronic hepatitis C. Moreover, miR-192 increased by $\mathrm{HCV}$ infection can directly upregulate TGF- $\beta 1$ expression, suggesting that it is also as a major factor mediating $\mathrm{HCV}$ infection-associated fibrogenesis [44].

\section{Wnt signaling}

Wnt is a highly conserved pathway and a key regulator of cell proliferation. Ad-HCV core adenovirus infection can downregulate miR-152 and upregulate the Wnt/ $\beta$ catenin effector Wnt1 in HepG2 cells. Notably, HCV downregulates miR-152 [45], which directly targets the WNT1 3'-UTR, thereby relieving the suppression and inducing Wnt1-mediated signaling that promotes in proliferation, G1-S transition, and colony formation in HepG2 cells. Nuclear localization of $\beta$-catenin-another marker of Wnt/ $\beta$-catenin activity-is also much higher in $\mathrm{HCV}$-infected and hepatocellular carcinoma patients than in healthy controls. For instance, miR-155 overexpression increases $\beta$-catenin nuclear localization in Huh7 cells [25], suggesting that it may regulate the 
immune response to $\mathrm{HCV}$ and thereby link $\mathrm{HCV}$ to cancer through Wnt $/ \beta$-catenin signaling.

\section{PI3K/Akt signaling}

Phosphatidylinositol 3-kinase and Akt/Protein Kinase B (PI3K/Akt) signaling is a classic and frequently engaged intracellular signal transduction pathway that mediates physiological and pathological processes-including metabolism, cell proliferation, cell survival, and angiogenesis in response to extracellular signals-via serine and/or threonine phosphorylation. miR-491, identified to be downregulated by HCV infection in Huh7 cell line, can enhance $\mathrm{HCV}$ replication both in $\mathrm{HCV}$ replicon cells and in cell culture-infectious HCV-infected cells. The study finds that the effect of miR-491 on HCV replication can be abolished by inhibiting PI3K, which indicates that augmentation of HCV replication by miR-491 is dependent on the PI3K/Akt pathway [46]. On the other hand, $\mathrm{HCV}$ also engages the PI3K pathway to activate miR-27, resulting in hepatic steatosis [47].

\section{Other signaling pathways}

Many other miRNAs have been predicted to regulate the NF- $\mathrm{kB}, \mathrm{MAPK}$, or other signaling during $\mathrm{HCV}$ infection. NF- $\mathrm{KB}$ is an important signaling pathway downstream of TNF- $\alpha$, which promotes inflammation, infection, immune processes, and cell apoptosis. It has been suggested that the non-structural protein $5 \mathrm{~A}$ (NS5A), encoded by HCV RNA genome, can decrease miR-503, which is abnormally expressed in various cancers and may play complicated and tissue-specific roles in cancer, and increase bcl-2 by inhibiting NF- $\mathrm{kB}$ activation [48]. This can be a potential mechanisms contributing to $\mathrm{HCV}$ infection, as NS5A plays a critical role in HCV replication. Moreover, miRNA-449a in liver biopsies is downregulated in chronic $\mathrm{HCV}$ infected patients, but not in patients with alcoholic or non-alcoholic liver disease, and the downregulation of miRNA-449a promotes TNFa-mediated activation of YKL40 through NOTCH signaling pathway [49]. On the other hand, miR-155 regulates IFNY production in natural killer cells via Tim-3 to balance immune clearance and immune injury during chronic hepatitis C [27].

\section{Conclusions}

$\mathrm{HCV}$ readily establishes chronic infection in adults. Virus genome factors, host innate immune response, and virus escape from the adaptive immune response are crucial to this process. Accordingly, clear understanding of the host immune response to $\mathrm{HCV}$ will illuminate specific

Table 1 miRNAs and the related immune responses and signaling pathways during hepatitis $C$ virus infection

\begin{tabular}{|c|c|c|c|}
\hline miRNAs & Related pathways & Immune responses and roles & References \\
\hline miR-122 & JAK/STAT signaling & $\begin{array}{l}\text { Stabilize the HCV RNA genome and stimulate virus replication } \\
\text { Predict response to therapy with IFN } \\
\text { Impede HCV entry into hepatocytes }\end{array}$ & {$[14-22]$} \\
\hline miR-155 & $\begin{array}{l}\text { Wnt signaling } \\
\text { Tim-3 signaling }\end{array}$ & $\begin{array}{l}\text { Enhance the development of inflammatory T-cells } \\
\text { Promote autoimmune inflammation } \\
\text { Regulate IFNr production from NK cells } \\
\text { Increase } \beta \text {-catenin nuclear localization in Huh7 cells }\end{array}$ & {$[23-27]$} \\
\hline miR-146 & TLR signaling & $\begin{array}{l}\text { Regulate the inflammatory and immune responses } \\
\text { Correlate with cholesterol metabolism }\end{array}$ & {$[28]$} \\
\hline miR-130a & JAK/STAT signaling & $\begin{array}{l}\text { Inhibit IFITM1 and the subsequent innate immune response } \\
\text { Play dual roles in HCV replication by shaping the host innate immune response }\end{array}$ & {$[29,30]$} \\
\hline miR-21 & $\begin{array}{l}\text { TLR signaling } \\
\text { TGF- } \beta 1 / S M A D s \text { signaling }\end{array}$ & $\begin{array}{l}\text { Repress the expression of type I IFN and the subsequent anti-viral response } \\
\text { Target SMAD7 } \\
\text { Stimulate the proliferation of hepatocellular carcinoma cells }\end{array}$ & {$[18,31,32]$} \\
\hline miR-181a & MAPK/ERK signaling & Decrease DUSP 6 expression and CD4 ${ }^{+} T$ cell dysfunction & {$[34]$} \\
\hline $\operatorname{miR}-27$ & PI3K/Akt signaling & $\begin{array}{l}\text { Inhibit virus infection and promotes lipid storage } \\
\text { Enable the virus to escape immune surveillance }\end{array}$ & {$[35,47]$} \\
\hline miR-196 & JAKVSTAT signaling & Suppress Bach1 expression, stimulates HMOX1 expression, and inhibits HCV gene expression. & {$[36-38]$} \\
\hline miR-125b & TLR signaling & Abolish the cytokine production & {$[39]$} \\
\hline miR-19a & JAK/STAT signaling & Enhance IFNa and interleukin-6 & {$[42]$} \\
\hline miR-192 & TGF- $\beta 1 / S M A D s$ signaling & $\begin{array}{l}\text { Upregulate TGF- } \beta 1 \text { expression } \\
\text { Mediate HCV infection-associated fibrogenesis }\end{array}$ & [44] \\
\hline miR-152 & Wnt signaling & $\begin{array}{l}\text { Target the WNT1 3'-UTR } \\
\text { Regulate proliferation, G1-S transition, and colony formation in HepG2 cells }\end{array}$ & {$[45]$} \\
\hline miR-491 & PI3K/Akt signaling & Enhance HCV replication & {$[46]$} \\
\hline $\operatorname{miR}-449 a$ & NOTCH signaling & Inhibit TNFa-mediated activation of YKL40 & {$[49]$} \\
\hline
\end{tabular}


mechanisms of pathogenesis, and promote the development of vaccines and immunotherapies. The body of evidence now suggests that miRNAs are key elements in $\mathrm{HCV}$ infection and the subsequent immune response, as well as in other physiological and pathological processes. Indeed, many miRNAs are dysregulated during $\mathrm{HCV}$ infection, and directly alter the host immune response or the replication of the virus by engaging various signaling pathways (Table 1). Contradictory miRNA activities have been described, highlighting complex roles in $\mathrm{HCV}$ infection and host immunity. Hence, specific roles and mechanisms require further verification, although these can now be predicted by bioinformatics. All the studies expressed demonstrate that miRNA expression is highly regulated in response to $\mathrm{HCV}$ infection and plays a significant role in virus infection and disease pathophysiology; however, the more detailed mechanisms in miRNA regulation and $\mathrm{HCV}$ infection, and disease pathophysiology still require further investigation.

\section{Abbreviations \\ HCV: hepatitis C virus; IFN: interferon; miRNA: microRNAs; DUSP: dual specific phosphatases; TLR: toll-like receptor; JAK/STAT: Janus kinase/signal transducer and activator of transcriptions; SOCS: suppressors of cytokine signaling; TGF- $\beta$ : transforming-growth factor- $\beta$; PI3K/Akt: phosphatidylinositol 3-kinase and Akt/ protein kinase B; NS5A: non-structural protein $5 \mathrm{~A}$.}

\section{Authors' contributions}

$\mathrm{HZ}$ made contributions to conception and design of the work, and was a major contributor in writing the manuscript. YG, QH and ML made contributions to conception of the work, and were involved in revising it critically for important intellectual content. All authors read and approved the final manuscript.

\section{Acknowledgements}

We are grateful to all members of the Clinical Laboratory of The Second Affiliated Hospital of Xi'an Jiaotong University for critically reading the manuscript. We thank Editage (https://www.editage.cn) for English language editing.

\section{Competing interests}

The authors declare that they have no competing interests.

\section{Availability of data and materials}

The datasets analysed during the current study are available in the NCBI repository, https://www.ncbi.nlm.nih.gov.

\section{Consent for publication}

All authors give their consents for publication.

\section{Ethics approval and consent to participate}

Not applicable.

\section{Funding}

Not applicable.

\section{Publisher's Note}

Springer Nature remains neutral with regard to jurisdictional claims in published maps and institutional affiliations.

Received: 5 November 2017 Accepted: 9 April 2018

Published online: 18 April 2018
References

1. Rehermann B, Nascimbeni M. Immunology of hepatitis B virus and hepatitis C virus infection. Nat Rev Immunol. 2005;5:215-29.

2. El-Serag HB. Epidemiology of viral hepatitis and hepatocellular carcinoma. Gastroenterology. 2012;142:1264-73.

3. Webster DP, Klenerman P, Dusheiko GM. Hepatitis C. Lancet. 2015:385:1124-35.

4. Jacobson IM, McHutchison JG, Dusheiko G, Di Bisceglie AM, Reddy KR, Bzowej $\mathrm{NH}$, et al. Telaprevir for previously untreated chronic hepatitis $\mathrm{C}$ virus infection. N Engl J Med. 2011;364:2405-16.

5. Feng J, Xing W, Xie L. Regulatory roles of microRNAs in diabetes. Int J Mol Sci. 2016;17:E1729.

6. Lindsay MA. microRNAs and the immune response. Trends Immunol. 2008;29:343-51.

7. Carthew RW, Sontheimer EJ. Origins and mechanisms of miRNAs and siRNAs. Cell. 2009;136:642-55.

8. Lee RC, Feinbaum RL, Ambros V. The C. elegans heterochronic gene lin-4 encodes small RNAs with antisense complementarity to lin-14. Cell. 1993;75:843-54.

9. Houbaviy HB, Murray MF, Sharp PA. Embryonic stem cell-specific microRNAs. Dev Cell. 2003;5:351-8.

10. Williams AE. Functional aspects of animal microRNAs. Cell Mol Life Sci. 2008;65:545-62.

11. Rehermann B. Hepatitis $C$ virus versus innate and adaptive immune responses: a tale of coevolution and coexistence. J Clin Invest. 2009;119:1745-54.

12. Verma P, Pandey RK, Prajapati P, Prajapati VK. Circulating microRNAs: potential and emerging biomarkers for diagnosis of human infectious diseases. Front Microbiol. 2016;7:1274.

13. Ura S, Honda M, Yamashita T, Ueda T, Takatori H, Nishino R, et al. Differential microRNA expression between hepatitis B and hepatitis $C$ leading disease progression to hepatocellular carcinoma. Hepatology. 2009:49:1098-112.

14. Jopling CL, Yi M, Lancaster AM, Lemon SM, Sarnow P. Modulation of hepatitis $C$ virus RNA abundance by a liver-specific microRNA. Science. 2005;309:1577-81.

15. García-Sastre A, Evans MJ. miR-122 is more than a shield for the hepatitis C virus genome. Proc Natl Acad Sci USA. 2013;110:1571-2.

16. Zhang C, Huys A, Thibault PA, Wilson JA. Requirements for human Dicer and TRBP in microRNA-122 regulation of HCV translation and RNA abundance. Virology. 2012;433:479-88.

17. Shan Y, Zheng J, Lambrecht RW, Bonkovsky HL. Reciprocal effects of micro-RNA-122 on expression of heme oxygenase- 1 and hepatitis $C$ virus genes in human hepatocytes. Gastroenterology. 2007;133:1166-74.

18. Marquez RT, Bandyopadhyay S, Wendlandt EB, Keck K, Hoffer BA, Icardi MS, et al. Correlation between microRNA expression levels and clinical parameters associated with chronic hepatitis $\mathrm{C}$ viral infection in humans. Lab Invest. 2010;90:1727-36.

19. Sarasin-Filipowicz M, Krol J, Markiewicz I, Heim MH, Filipowicz W. Decreased levels of microRNA miR-122 in individuals with hepatitis $C$ responding poorly to interferon therapy. Nat Med. 2009;15:31-3.

20. Sendi H, Mehrab-Mohseni M, Foureau DM, Ghosh S, Walling TL, Steuerwald N, et al. miR-122 decreases HCV entry into hepatocytes through binding to the 3' UTR of OCLN mRNA. Liver Int. 2015;35:1315-23.

21. Varnholt H, Drebber U, Schulze F, Wedemeyer I, Schirmacher P, Dienes HP, et al. microRNA gene expression profile of hepatitis $C$ virus-associated hepatocellular carcinoma. Hepatology. 2008;47:1223-32.

22. Kutay H, Bai S, Datta J, Motiwala T, Pogribny I, Frankel W, et al. Downregulation of miR-122 in the rodent and human hepatocellular carcinomas. J Cell Biochem. 2006;99:671-8.

23. O'Connell RM, Kahn D, Gibson WS, Round JL, Scholz RL, Chaudhuri AA, et al. microRNA-155 promotes autoimmune inflammation by enhancing inflammatory T cell development. Immunity. 2010;33:607-19.

24. Jiang M, Broering R, Trippler M, Wu J, Zhang E, Zhang $X$, et al. microRNA-155 controls Toll-like receptor 3 - and hepatitis C virus-induced immune responses in the liver. JViral Hepat. 2014;21:99-110.

25. Zhang Y, Wei W, Cheng N, Wang K, Li B, Jiang X, et al. Hepatitis C virusinduced up-regulation of microRNA-155 promotes hepatocarcinogenesis by activating Wnt signaling. Hepatology. 2012;56:1631-40. 
26. Bala S, Tilahun Y, Taha O, Alao H, Kodys K, Catalano D, et al. Increased microRNA-155 expression in the serum and peripheral monocytes in chronic HCV infection. J Transl Med. 2012;10:151.

27. Cheng YQ, Ren JP, Zhao J, Wang JM, Zhou Y, Li GY, et al. microRNA-155 regulates interferon-gamma production in natural killer cells via Tim-3 signalling in chronic hepatitis C virus infection. Immunology. 2015; 145:485-97.

28. Sidorkiewicz M, Grek M, Jozwiak B, Krol A, Piekarska A. The impact of chronic hepatitis $C$ infection on cholesterol metabolism in PBMCs is associated with microRNA-146a expression. Eur J Clin Microbiol Infect Dis. 2017:36:697-702.

29. Bhanja Chowdhury J, Shrivastava S, Steele R, Di Bisceglie AM, Ray R, Ray RB. Hepatitis C virus infection modulates expression of interferon stimulatory gene IFITM1 by upregulating miR-130A. J Virol. 2012;86:10221-5.

30. Li S, Duan X, Li Y, Liu B, MCGilvray I, Chen L. microRNA-130a inhibits HCV replication by restoring the innate immune response. J Viral Hepat. 2014;21:121-8.

31. Chen Y, Chen J, Wang H, Shi J, Wu K, Liu S, et al. HCV-induced miR-21 contributes to evasion of host immune system by targeting MyD88 and IRAK1. PLoS Pathog. 2013;9:e1003248.

32. Ladeiro Y, Couchy G, Balabaud C, Bioulac-Sage P, Pelletier L, Rebouissou $S$, et al. microRNA profiling in hepatocellular tumors is associated with clinical features and oncogene/tumor suppressor gene mutations. Hepatology. 2008:47:1955-63.

33. Li G, Yu M, Lee WW, Tsang M, Krishnan E, Weyand CM, et al. Decline in miR-181a expression with age impairs $T$ cell receptor sensitivity by increasing DUSP6 activity. Nat Med. 2012;18:1518-24.

34. Li GY, Zhou Y, Ying RS, Shi L, Cheng YQ, Ren JP, et al. Hepatitis C virusinduced reduction in miR-181a impairs CD4(+) T-cell responses through overexpression of DUSP6. Hepatology. 2015;61:1163-73.

35. Shirasaki T, Honda M, Shimakami T, Horii R, Yamashita T, Sakai Y, et al. microRNA-27a regulates lipid metabolism and inhibits hepatitis C virus replication in human hepatoma cells. J Virol. 2013;87:5270-86.

36. Hou W, Tian Q, Zheng J, Bonkovsky HL. microRNA-196 represses Bach1 protein and hepatitis $C$ virus gene expression in human hepatoma cells expressing hepatitis C viral proteins. Hepatology. 2010;51:1494-504.

37. Liu B, Xiang Y, Zhang HS. Circulating microRNA-196a as a candidate diagnostic biomarker for chronic hepatitis C. Mol Med Rep. 2015;12:105-10.

38. Pedersen IM, Cheng G, Wieland S, Volinia S, Croce CM, Chisari FV, et al. Interferon modulation of cellular microRNAs as an antiviral mechanism. Nature. 2007;449:919-22.
39. Peng C, Wang H, Zhang WJ, Jie SH, Tong QX, Lu MJ, et al. Inhibitory effect of miR-125b on hepatitis C virus core protein-induced TLR2/MyD88 signaling in THP-1 cells. World J Gastroenterol. 2016;22:4354-61.

40. Yoshikawa T, Takata A, Otsuka M, Kishikawa T, Kojima K, Yoshida H, et al. Silencing of microRNA-122 enhances interferon-a signaling in the liver through regulating SOCS3 promoter methylation. Sci Rep. 2012;2:637.

41. Yang X, Marcucci K, Anguela X, Couto LB. Preclinical evaluation of an anti-HCV miRNA cluster for treatment of HCV infection. Mol Ther. 2013;21:588-601.

42. Collins AS, McCoy CE, Lloyd AT, O'Farrelly C, Stevenson NJ. miR-19a: an effective regulator of SOCS3 and enhancer of JAK-STAT signalling. PLOS ONE. 2013;8:e69090.

43. Mukherjee A, Di Bisceglie AM, Ray RB. Hepatitis C virus-mediated enhancement of microRNA miR-373 impairs the JAK/STAT signaling pathway. J Virol. 2015;89:3356-65.

44. Kim JH, Lee $\mathrm{CH}$, Lee $\mathrm{SW}$. Hepatitis C virus infection stimulates transforming growth factor- $\beta 1$ expression through up-regulating miR-192. J Microbiol. 2016:54:520-6.

45. Huang S, Xie Y, Yang P, Chen P, Zhang L. HCV core protein-induced down-regulation of microRNA-152 promoted aberrant proliferation by regulating Wnt1 in HepG2 cells. PLoS ONE. 2014;9:e81730.

46. Ishida H, Tatsumi T, Hosui A, Nawa T, Kodama T, Shimizu S, et al. Alterations in microRNA expression profile in HCV-infected hepatoma cells: involvement of miR-491 in regulation of HCV replication via the PI3 kinase/Akt pathway. Biochem Biophys Res Commun. 2011;412:92-7.

47. Singaravelu R, Chen R, Lyn RK, Jones DM, O'Hara S, Rouleau Y, et al. Hepatitis $C$ virus induced up-regulation of microRNA-27: a novel mechanism for hepatic steatosis. Hepatology. 2014;59:98-108.

48. Xie Z, Xiao Z, Wang F. Hepatitis C virus nonstructural 5A protein (HCVNS5A) inhibits hepatocyte apoptosis through the NF-kb/miR-503/bcl-2 pathway. Mol Cells. 2017;40:202-10.

49. Sarma NJ, Tiriveedhi V, Subramanian V, Shenoy S, Crippin JS, Chapman WC, et al. Hepatitis C virus mediated changes in miRNA-449a modulates inflammatory biomarker YKL40 through components of the NOTCH signaling pathway. PLOS ONE. 2012;7:e50826.
Ready to submit your research? Choose BMC and benefit from:

- fast, convenient online submission

- thorough peer review by experienced researchers in your field

- rapid publication on acceptance

- support for research data, including large and complex data types

- gold Open Access which fosters wider collaboration and increased citations

- maximum visibility for your research: over $100 \mathrm{M}$ website views per year

At BMC, research is always in progress.

Learn more biomedcentral.com/submissions 